\title{
Lógica difusa vs. modelo de regresión múltiple para la selección de personal
}

\author{
Fuzzy logic vs. multiple regression for selection personnel \\ Carlos A. Díaz-Contreras ${ }^{1} \quad$ Alejandra Aguilera-Rojas ${ }^{1} \quad$ Nathaly Guillén-Barrientos $^{1}$ \\ Recibido 21 de diciembre de 2012, aceptado 23 de mayo de 2014 \\ Received: December 21, 2012 Accepted: May 23, 2014
}

\begin{abstract}
RESUMEN
La incorporación de nuevo personal o la reasignación del ya existente a tareas específicas constituyen una decisión importante, porque el acierto en ella determinará la propia supervivencia de la empresa. En este contexto se vuelve relevante contar con un modelo de selección de personal que considere la información ambigua y los grados de incertidumbre que están asociados al momento de evaluar las valoraciones cualitativas de los postulantes y que pueda entregar resultados certeros y precisos, garantizando de esta manera el buen desempeño del cargo y reduciendo así el riesgo que conlleva la incorporación de nuevas personas.

En este trabajo se elaboró un modelo de selección de personal, en condiciones de incertidumbre, aplicando Lógica Difusa, utilizando como datos de entrada las descripciones de cargos de una empresa del retail, con variables difusas triangulares y con solapamiento. Este fue comparado con un modelo clásico de regresión múltiple.

Los resultados mostraron que, en este caso, el uso del modelo de regresión múltiple es más eficiente que el modelo de lógica difusa optado.
\end{abstract}

Palabras clave: Lógica difusa, selección de personal, recurso humano, regresión múltiple, desempeño del trabajador.

\begin{abstract}
The incorporation of new personnel or the reallocation of existing tasks is an important decision, since its correctness will determine the survival of the company. In this context, having a model of personnel selection, that considers the associated ambiguous information and degrees of uncertainty, becomes relevant when assessing the qualitative value of the applicants, able to deliver accurate and precise results thus ensuring the good performance of the position and reducing the associated risk with the incorporation of new people. In this work, a model of personnel selection, in conditions of uncertainty using fuzzy logic and having as input the data descriptions of positions of a retail industry, with triangular fuzzy variables and overlap was developed. This was compared with a classical model of multiple regressions. The results showed in this case, that the use of the model of multiple regressions is more efficient than the opted model of fuzzy logic.
\end{abstract}

Keywords: Fuzzy logic, human resources, selection of personnel, multiple regressions, worker perfomance.

\section{INTRODUCCIÓN}

La incorporación de nuevo personal o la reasignación del ya existente a tareas específicas constituyen una decisión importante, porque el acierto en ella determinará la propia supervivencia de la empresa. Esto es aplicable a cualquier sector, pero cobra mayor relevancia en aquellos mercados globales altamente turbulentos o competentes, en que desarrollan actividades y precisan contar con

\footnotetext{
1 Escuela Universitaria de Ingeniería Industrial, Informática y de Sistemas. Universidad de Tarapacá. 18 de Septiembre 2222. Arica, Chile.E-mail: cdiazc@uta.cl; alejandra.m.aguilera@gmail.com; nguillen@parinacoop.cl
} 
recursos humanos que estén muy bien dotados de flexibilidad y adaptabilidad suficientes, de ahí que una correcta selección del personal impactará en un alto grado en el desarrollo futuro de la empresa.

Al modelo clásico de regresión múltiple para evaluar y seleccionar al mejor postulante a un puesto se propone como alternativa un modelo basado en lógica difusa, que permita tanto minimizar los riesgos derivados de la realización de tareas por personal inadecuado como maximizar la utilidad de la empresa con la ubicación óptima de los trabajadores, permitiendo incorporar en ella toda la información de la que se dispone por ambigua o subjetiva que esta sea, así como con las imprecisiones que este tipo de toma de decisiones conlleva.

El objetivo general de este trabajo es evaluar cuál de los modelos (regresión múltiple o lógica difusa) entrega resultados más certeros al momento de evaluar y elegir un postulante para un puesto específico.

\section{GESTIÓN DEL RECURSO HUMANO}

Una empresa cuenta con diversos tipos de recursos que le permiten funcionar óptimamente y alcanzar sus metas propuestas, el que está conformado por los empleados, trabajadores, colaboradores y demás personas que integran una organización, siendo entonces el Departamento de Personal o Departamento de Recursos Humanos el área de la empresa que se dedica a organizar, desarrollar y poner en funcionamiento a estas personas, para conseguir en forma eficiente y eficaz los objetivos de la organización, utilizando para ello una serie de funciones y actividades, como es la selección, contratación, formación, empleo y retención de los trabajadores [17].

La Gestión de Recursos Humanos se puede definir como "la ciencia y la práctica que se ocupan de la naturaleza de las relaciones de empleo y del conjunto de decisiones, acciones y cuestiones vinculadas a dichas relaciones" [10]. Entonces, la gestión integral de recursos humanos se basa en cinco pilares fundamentales, estos son [17]:

Diseño de la estructura u organización del trabajo: Se realizan análisis y descripciones de puestos, valoración de la política salarial y de las relaciones laborales.
Selección y contratación: Se refiere a la detección de necesidades, reclutamiento, selección, contratación, incorporación e integración (planes de acogida) de las personas a la empresa.

Evaluación: Consiste en la dirección por objetivos, evaluación del desempeño y evaluación por competencias.

Mejorar: Busca mejorar el clima laboral, la motivación, los planes de formación, la adecuación puesto-persona, la promoción y los planes de carrera dentro de una organización.

Revisión: Se reestructuran las plantillas y se revisan los objetivos y el modelo de gestión. Es importante que haya retroalimentación para poder evaluar los resultados y los objetivos.

Por otro lado, actualmente todas las organizaciones viven en un constante proceso de transformación que afecta también al personal de la empresa, esta sufre frecuentes modificaciones, lo que genera la necesidad de proveer en forma permanente nuevos empleados. Por tanto, es conveniente que la empresa tenga siempre abierto un proceso de reclutamiento y de selección de recursos humanos para el mantenimiento de su personal [19].

Selección de personal: Consiste en elegir a una persona para un puesto de trabajo con un determinado perfil, susceptible de ser definido mediante medidas que permitan ser comparadas con las cualidades del candidato. El proceso de selección se puede resumir en las siguientes tres fases [9]:

\section{a) Establecimiento del perfil del puesto de trabajo:} Se realiza por medio del análisis de las tareas encomendadas con posibilidades objetivas para su realización. El perfil comprende también la enumeración de las cualidades que el candidato debe poseer para la correcta realización de las actividades que el puesto de trabajo comporta, así como el grado de importancia que dicha competencia tiene en relación con tal puesto.

b) Evaluación del candidato: Se dispone de una amplia gama de posibilidades para elegir las pruebas (formularios, entrevistas, exámenes, tests, etc.) que, de alguna manera, intentan determinar los niveles de aptitud de una persona en relación 
con ciertas cualidades que se estiman precisas para desarrollar correctamente las tareas de un puesto de trabajo.

c) Adaptación del candidato al perfil: Una vez conocidos los niveles en que cada postulante posee una determinada cualidad se procede a su comparación con las cualidades del perfil del puesto de trabajo establecido, lo que permite conocer el grado de adaptación de cada candidato y obtener, en definitiva, un orden de preferencia entre ellos.

En el proceso de selección se debe contemplar además del perfil profesional de los candidatos, los requerimientos del puesto de trabajo y la cultura de la organización para que la selección garantice el desempeño correcto del puesto y reduzca el riesgo que conlleva incorporar a nuevas personas a la empresa [17].

\section{LÓGICA DIFUSA}

La Lógica Difusa es un formalismo matemático que pretende emular la habilidad que tienen algunas personas para tomar decisiones correctas a partir de datos vagos o imprecisos y que están expresados lingüísticamente. Permite tratar información imprecisa, como estatura media o temperatura baja, en términos de conjuntos difusos que se combinan en reglas para definir acciones, por ejemplo, "si la temperatura es alta entonces enfriar mucho" [14].

Conjuntos difusos. Los conjuntos difusos pueden ser considerados como una generalización de los conjuntos clásicos: la teoría clásica de conjuntos solo contempla la pertenencia o no pertenencia de un elemento a un conjunto, sin embargo la teoría de conjuntos difusos contempla la pertenencia parcial de un elemento a un conjunto, es decir, cada elemento presenta un grado de pertenencia a un conjunto difuso que puede tomar cualquier valor entre $0 \mathrm{y}$ 1. Este grado de pertenencia se define mediante la función de pertenencia asociada al conjunto difuso: para cada valor que pueda tomar un elemento o variable de entrada $x$ la función de pertenencia $\mu_{A}(x)$ proporciona el grado de pertenencia de este valor de $x$ al conjunto difuso $A$ [15].

Función de pertenencia. La forma de la función de pertenencia utilizada depende del criterio aplicado en la resolución de cada problema y variará en función de la cultura, geografía, época o punto de vista del usuario. La única condición que debe cumplir una función de pertenencia es que tome valores entre 0 y 1 , con continuidad. Según [15], una de las funciones de pertenencia más utilizada por su simplicidad matemática es la triangular: definida por los límites $(\mathrm{a}, 0)$ y $(\mathrm{c}, 0)$ y por el punto central $(b, 1)$ tal que:

$$
\mu_{F}(x)= \begin{cases}0 & \text { si } x \leq a \\ \frac{x-a}{b-a} & \text { si } x \in(a, b] \\ \frac{c-x}{c-b} & \text { si } x \in[b, c) \\ 1 & \text { si } x \geq c\end{cases}
$$

Donde, $x=$ variable de entrada; $a=$ punto en el eje $\mathrm{x}$ que señala el primer vértice del triángulo; $b=$ punto en el eje $\mathrm{x}$ que señala el segundo vértice del triángulo; $c=$ punto en el eje x que señala el tercer vértice del triángulo.

Una misma variable puede estar asociada a varias funciones de pertenencias a la vez, y estas pueden estar solapadas, es decir, "el vaso puede estar medio lleno y medio vacío a la vez". Por ejemplo, en la Figura 1 el grupo de trabajadores "Mala" está constituido por todos los que tienen entre $0 \mathrm{y}$ 4 años de experiencia laboral y el grupo "Regular" lo conforman todos aquellos que tienen entre 2 y 6 años de experiencia laboral. Por lo tanto, el trabajador con una experiencia de 3,5 años tendrá una pertenencia de 0,25 en el primer grupo, y una pertenencia de 0,75 en el segundo grupo.

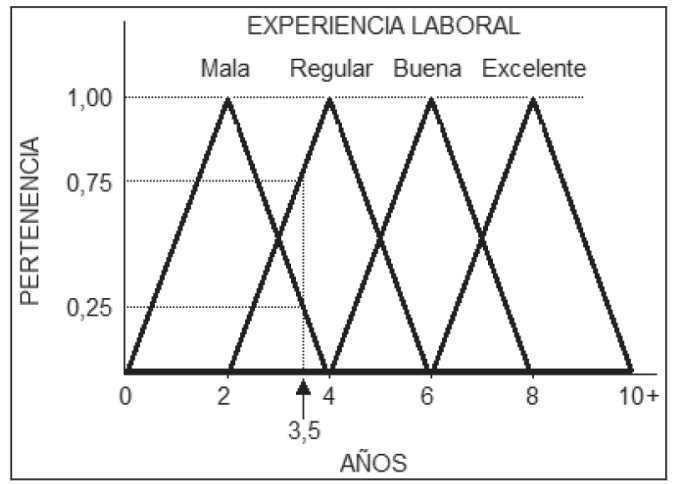

Figura 1. Funciones de pertenencias triangulares con solapamiento para la "experiencia laboral". 
Operaciones con conjuntos difusos. Las operaciones básicas entre conjuntos difusos son:

a) Unión (OR): La unión de dos conjuntos difusos A y $\mathrm{B}$ es un conjunto difuso A $\mathrm{U} B$ en $\mathrm{U}$ cuya función de pertenencia es:

$$
\mu_{A \cup B}(x)=\operatorname{máx}\left[\mu_{A}(x), \mu_{B}(x)\right]
$$

b) Intersección ( $A N D$ ): La intersección de dos conjuntos difusos $\mathrm{A}$ y $\mathrm{B}$ es un conjunto difuso $\mathrm{A} \cap \mathrm{B}$ en $\mathrm{U}$ con función de pertenencia:

$$
\mu_{A \cap B}(x)=\min \left[\mu_{A}(x), \mu_{B}(x)\right]
$$

Las funciones que definen la unión y la intersección de conjuntos difusos pueden generalizarse, a condición de cumplir ciertas restricciones. Las funciones que cumplen estas condiciones se conocen como Conorma Triangular (T-Conorma) y Norma Triangular (T-Norma). En la mayoría de las aplicaciones a la ingeniería de la lógica difusa se usan como T-Conorma el operador máximo y como T-Norma el operador mínimo.

Variable lingüística. Se denomina Variable Lingüística a aquella que puede tomar por valor algún término del lenguaje natural, como "mucho", "poco", "positivo", etc., que son palabras que desempeñan el papel de etiquetas en un conjunto difuso [15].

Sistemas de lógica difusa. Se pueden distinguir tres clases de sistemas basados en lógica difusa de acuerdo con la forma de las reglas y el tipo de entradas y salidas [1]:

a) Sistemas difusos tipo Mamdani: Están compuestos por una base de conocimiento, un motor de inferencias, un bloque de fusificación y un bloque de desfusificación. Las reglas que manejan esta clase de sistemas son de la forma: $\operatorname{Si} X_{1}$ es $A_{1}$ y $X_{2}$ es $A_{2}$ y $\ldots X_{n}$ es $A_{n}$, entonces, $\mathrm{Y}$ es B. Donde las entradas $X_{i}$ y la salida $Y$ son números (no borrosos) y por lo tanto, los $A_{i}$ y B son conjuntos borrosos sin interpretación directa.

b) Sistemas difusos puros: Estos sistemas tienen como entrada y como salida conjuntos borrosos. $\mathrm{Al}$ no realizar ninguna transformación sobre las entradas o sobre las salidas, tienen solo dos componentes principales: una base de conocimiento y un motor de inferencias. Las reglas lingüísticas empleadas son de la forma: Si $X_{1}$ es $A_{1}$ y $X_{2}$ es $A_{2}$ y $\ldots X_{n}$ es $A_{n}$, entonces, $\mathrm{Y}$ es B. Donde $X_{i}$ e $\mathrm{Y}$ son variables lingüísticas, y los $A_{i}$ y $\mathrm{B}$ son etiquetas lingüísticas asociadas a conjuntos difusos.

c) Sistemas difusos tipo Takagi-Sugeno-Kang: En lugar de trabajar con reglas linguiísticas, Takagi, Sugeno y Kang propusieron un nuevo modelo basado en reglas donde el antecedente estaba compuesto de variables lingüísticas y el consecuente se representaba como una función de las variables de entrada [21]. La forma más habitual de esta clase de reglas es la siguiente: $\operatorname{Si} X_{1}$ es $A_{1}$ y $\ldots X_{n}$ es $A_{n}$, entonces, $Y=p_{1} X_{1}+\ldots+p_{n} X_{n}+p_{0}$. Siendo $X_{i}$ las variables de entrada, $\mathrm{Y}$ la variable de salida, y $p_{i}$ parámetros reales.

Superficies de implicación. Según [23], para que se cumpla la equivalencia entre lógica clásica y lógica difusa se debe traducir por una t-norma, ya que preserva la relación causa-efecto y el sentido físico. Cada regla define una superficie de implicación: para cada posible valor del antecedente se puede obtener el conjunto difuso que resulta de realizar la implicación; la superposición de todos estos conjuntos difusos forman la superficie de implicación de esa regla.

Agregación lógica. Una vez evaluadas todas las reglas y obtenidos los conjuntos difusos de salida modificados, hay que realizar la agregación de todas las reglas para obtener un resultado único de la actuación de todas ellas. Esta agregación es una unión lógica, y para conservar la equivalencia entre lógica clásica y lógica difusa se traduce por una T-Conorma.

Métodos de desfusificación. Según [20], aunque existen distintos métodos de desfusificación, uno de los más usados en aplicaciones en gestión es el Centro de Sumas. Este considera la contribución individual del área de cada conjunto de salida formado al aplicar cada una de las reglas:

$$
x_{\text {salida }}=\frac{\sum_{i=1}^{l} x_{i} \sum_{k=1}^{n} \mu^{(k)}\left(x_{i}\right)}{\sum_{i=1}^{l} \sum_{k=1}^{n} \mu^{(k)}\left(x_{i}\right)}
$$


Donde, $x_{\text {salida }}$ es la coordenada del centro de sumas, $x_{i}$ son los distintos valores que puede tomar $x$ y $\mu^{(k)}$ representa el conjunto difuso obtenido al aplicar el procedimiento de inferencia.

\section{METODOLOGÍA}

Planteamiento del problema. Debido a que se requiere evaluar cuál de los dos modelos entrega mejores resultados al momento de evaluar los postulantes para un puesto se seguirá el siguiente procedimiento:

- Para cada puesto de trabajo, y usando las fichas de postulaciones, se aplicarán ambos modelos a todos los trabajadores que están desempeñando un determinado cargo (observación: el ingreso de esos trabajadores a la empresa ha ocurrido en distintas fechas).

- En cada modelo, y por cada puesto de trabajo, se conocerá cuál es el mejor postulante (observación: en este caso correspondería al "mejor trabajador hipotético").

- Usando la última evaluación anual aplicada a cada uno de los trabajadores se conocerá cuál es el "mejor trabajador real" por cada puesto.

- Y por último, en cada puesto de trabajo, conociendo el "mejor trabajador real" y el "mejor trabajador hipotético", se podrá evaluar cuál de los dos modelos tuvo más acierto.

Datos. Se utilizará como datos de entrada la información de los procesos de selección de personal del supermercado LIDER de Walmart Chile ubicado en Diego Portales, Arica, Chile.

Solo se considerará el proceso de selección para el cargo de Colaborador y no se incluirá el proceso de selección para los cargos de gerente de venta, subadministradores y jefes de las áreas de la empresa, ya que no fue posible acceder a sus evaluaciones, estas son tratadas de manera confidencial en la central de Santiago. Con ello, la muestra a estudiar se limita a los Colaboradores que trabajan actualmente en el supermercado 45 horas semanales en las secciones de: "Seguridad", "Hardline", "Frutas y Verduras", "Fiambrería y Deli", "Entretenimiento", "Consumible", "Carnes y Pescados", "Panadería y Pastelería" y "Vestuario y Mundo Bebé". O sea, 70 trabajadores:
Tabla 1. Cantidad de colaboradores por Sección.

\begin{tabular}{|c|c|}
\hline Sección & $\mathbf{N}^{0}$ de Colaboradores \\
\hline Seguridad & 20 \\
\hline Hardlines & 6 \\
\hline Frutas y Verduras & 3 \\
\hline Fiambrería y Deli & 3 \\
\hline Entretenimiento & 8 \\
\hline Consumible & 5 \\
\hline Carnes y Pescados & 5 \\
\hline Panadería y Pastelería & 12 \\
\hline Vestuario y Mundo bebé & 8 \\
\hline Total & 70 \\
\hline
\end{tabular}

Se obtuvieron los resultados de las entrevistas de trabajo realizadas por los jefes de cada sección a los postulantes a algún cargo de "Colaborador" (único instrumento que utiliza la empresa para seleccionar a los futuros "Colaboradores"). Los aspectos que se evalúan son los requisitos o exigencias particulares que el "Colaborador" debe poseer para el buen desempeño laboral en una determinada sección. Entre estos requisitos se distinguen aquellos que son exigidos en forma obligatoria para todas las secciones, estos son, que la persona sea mayor de edad (mayor de 18 años) y que su nivel mínimo de educación sea la enseñanza media (por ejemplo, para el caso de la sección "Seguridad" se exige, además de lo anterior, que posean una certificación médica que cerciore un buen estado físico, que no se encuentren en Dicom, y en el caso de los varones, que estén al día con el servicio militar).

Selección del mejor postulante. Si consideramos un puesto de trabajo para el que son necesarias $n$ competencias, que expresamos como un conjunto finito de características exigidas $\mathrm{X}=\{c 1, c 2, \ldots$, $c$ n $\}$, y disponemos de $R$ postulantes, Post $=\{P 1$, $P 2, \ldots, P \mathrm{R}\}$, para cubrir las vacantes, la selección se debe hacer evaluando a cada postulante en las $n$ competencias. Esta evaluación puede entenderse como el grado de pertenencia a un conjunto difuso o $\varphi_{\text {-difuso }}$. [6]

Tal como se muestra en la Figura 2, los $R$ postulantes deben ser valorados en las $n$ competencias, además, Recursos Humanos debe definir un candidato ideal, prefijando las valoraciones que considera óptimas en cada competencia. Luego, al comparar ambos, se puede determinar la distancia respecto del candidato 
ideal. En cualquier caso, se puede asegurar que un aspirante al puesto será mejor para la empresa cuanto más "parecido" sea al candidato ideal prefijado.

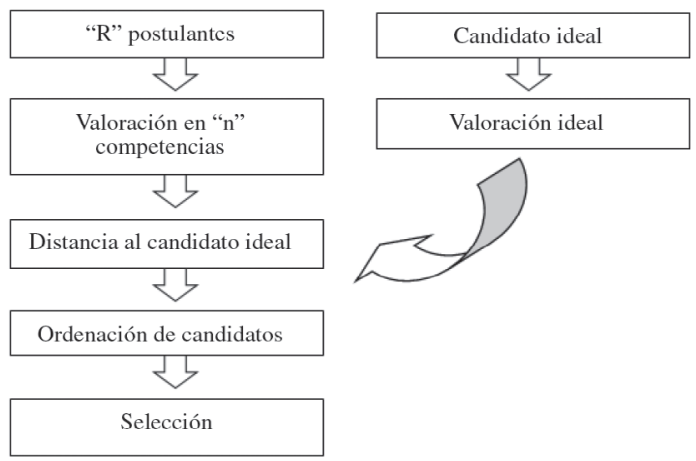

Figura 2. Esquema general a ocupar para la selección de personal.

De acuerdo con [12], la mejor forma de determinar el parecido con el candidato ideal es mediante el cálculo de la "Distancia de Hamming" o el "Coeficiente de Adecuación".

Dados un conjunto de referencia $X=\left(x_{1}, x_{2}, x_{3}\right.$, $\left.\ldots, x_{n}\right)$ y dos conjuntos $\varphi_{\text {-difuso }} \tilde{A}^{\varphi}, \tilde{B}^{\varphi}$, con funciones de pertenencia $\mu_{\tilde{A}}^{\varphi}\left(x_{j}\right)=\left[a_{x_{j}}^{1}, a_{x_{j}}^{2}\right]$ y $\mu_{\tilde{B}}^{\varphi}\left(x_{j}\right)=\left[b_{x_{j}}^{1}, b_{x_{j}}^{2}\right]$, con $\mathrm{j}=1,2,3, \ldots, \mathrm{n}$, se define:

a) La Distancia de Hamming como:

$$
\begin{aligned}
& d\left(\tilde{A}^{\varphi}, \tilde{B}^{\varphi}\right)=\frac{1}{2 n}\left(\sum_{j=1}^{n}\left|\mu_{\tilde{A}}^{\varphi}\left(x_{j}\right)-\mu_{\tilde{B}}^{\varphi}\left(x_{j}\right)\right|\right)= \\
& \frac{1}{2 n}\left(\sum_{j=1}^{n}\left(\left|a_{x_{j}}^{1}-b_{x_{j}}^{1}\right|+\left|a_{x_{j}}^{2}-b_{x_{j}}^{2}\right|\right)\right)
\end{aligned}
$$

b) El Coeficiente de Adecuación como:

$$
\mu_{\tilde{B}^{\varphi}}\left(\tilde{A}^{\varphi}\right)=\frac{1}{n} \sum_{j=1}^{n} \mu_{B^{\varphi}}^{x_{j}}\left(\tilde{A}^{\varphi}\right)
$$

Donde,

$$
\mu_{\tilde{B}^{\varphi}}^{x_{j}}\left(\tilde{A}^{\varphi}\right)= \begin{cases}1 & \text { si }\left[b_{x_{j}}^{1}, b_{x_{j}}^{2}\right] \subseteq\left[a_{x_{j}}^{1}, a_{x_{j}}^{2}\right] \\ \frac{\text { longitud }\left(\left[b_{x_{j}}^{1}, b_{x_{j}}^{2}\right] \cap\left[a_{x_{j}}^{1}, a_{x_{j}}^{2}\right]\right)}{\operatorname{longitud}\left(\left[b_{x_{j}}^{1}, b_{x_{j}}^{2}\right] \cup\left[a_{x_{j}}^{1}, a_{x_{j}}^{2}\right]\right)} & \text { si }\left[b_{x_{j}}^{1}, b_{x_{j}}^{2}\right] \notin\left[a_{x_{j}}^{1}, a_{x_{j}}^{2}\right]\end{cases}
$$

La Distancia de Hamming calcula la diferencia entre los extremos de los intervalos, por ello, no diferencia entre un exceso o un defecto respecto del ideal, por lo que evalúa ambos de forma equivalente. En cambio, el Coeficiente de Adecuación incluye implícitamente una corrección de los excesos y defectos.

Intervalos. Si la valoración de las competencias se basa en intervalos, ya que ella se acerca más al pensamiento humano, para poder trabajar con intervalos se necesita introducir una relación de orden entre ellos [6]: Dados los intervalos $A=\left[a_{1}, a_{2}\right], B\left[b_{1}, b_{2}\right] \subset R$, decimos que A es mayor que $\mathrm{B}$ si y solo si:

$$
\begin{cases}k_{1} a_{1}+k_{2} a_{2} \succ k_{1} b_{1}+k_{2} b_{2} & \text { con } k_{1} a_{1}+k_{2} a_{2} \neq k_{1} b_{1}+k_{2} b_{2} \\ a_{1} \succ b_{1} & \text { con } k_{1} a_{1}+k_{2} a_{2}=k_{1} b_{1}+k_{2} b_{2}\end{cases}
$$

Donde, $k_{1}$ y $k_{2}$ son dos constantes positivas fijadas a priori.

Aplicación de la lógica difusa. La aplicación consta de seis pasos:

Paso 1: Definir los requisitos exigidos por cada tipo de puesto. Estos a su vez están divididos en:

$\stackrel{\leftrightarrow}{\rightarrow}$ Requisitos críticos: Estos requisitos deben cumplirse de manera obligatoria, previa a la evaluación del postulante. Si no los cumple no está dentro del perfil deseado (método no compensatorio), resultando ser "No Apto" para dicho puesto.

$\stackrel{\leftrightarrow}{\rightarrow}$ Requisitos propios del puesto: Estos requisitos son deseables que los cumpla el postulante, y puede compensar el sobrecumplimiento de algunos de ellos con déficit en otros. Siguiendo la metodología de [7] y [4], se señala la etiqueta lingüística apropiada para cada requisito: "Excelente", "Bueno", "Regular" y "Malo".

Paso 2: Definir los coeficientes. Se debe definir la importancia relativa que tendrá cada grupo de requisitos propios del puesto dentro del total.

Paso 3: Ingresar los antecedentes del postulante. Se deben ingresar los requisitos obligatorios y las etiquetas lingüísticas para cada requisito propio del puesto. 
Paso 4: Definir las variables difusas. Se debe definir los rangos de valores de las variables difusas tanto para el candidato ideal como para cada uno de los postulantes. Se usarán cuatro intervalos que corresponden a variables difusas triangulares y con solapamiento: Excelente, intervalo $(6,10)$; Bueno, intervalo $(4,8)$; Regular, intervalo $(2,6)$ y Malo, intervalo $(0,4)$.

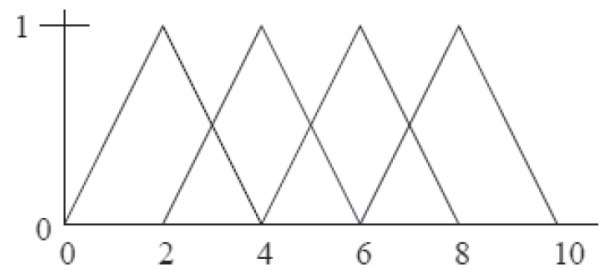

Figura 3. Variables difusas triangulares y con solapamiento.

Paso 5: Calcular la calificación por grupo de requisitos propios del puesto. En cada uno de los grupos de requisitos propios del puesto, tanto del candidato ideal como de los postulantes, se deben calcular los promedios de los inicios de los intervalos, de los centros de los intervalos y de los finales de los intervalos de las etiquetas lingüísticas.

Paso 6: Elegir al postulante. Considerando las calificaciones por requisitos propios del puesto (Paso 5) y la definición de los coeficientes (Paso 2) para cada postulante se calcula tanto la Distancia de Hamming como el Coeficiente de Adecuación, y se elige el mejor postulante para el puesto.

\section{Aplicación del modelo clásico de regresión} múltiple. Permitirá seleccionar al candidato que presenta el mayor valor al utilizar varios predictores. Este modelo asume que la variable dependiente tiene una distribución normal, y que existen correlaciones entre los predictores que pueden compensarse entre sí, donde cada uno de los predictores $(X)$ guarda correlaciones lineales con el criterio $(Y)$. Por esta razón, este modelo también podría ser considerado como de "compensación lineal". [16]

Los pasos a seguir según este método son cinco y se detallan a continuación [8]:

Paso 1: Análisis del puesto de trabajo. Se debe realizar un examen de los puestos de trabajo que se desean cubrir, determinando para ello las características cognitivas y de personalidad requeridas para desempeñar el cargo.

Paso 2: Selección de criterios y predictores. El criterio o la variable dependiente $(Y)$ estará constituida por el desempeño laboral, que será medido como el "Potencial para el cargo" y los predictores o las variables independientes $\left(X_{1}, X_{2}, \ldots, X_{n}\right)$ serán los resultados obtenidos por los sujetos en las entrevistas de trabajos.

Paso 3: Medición de criterio y predictores. Una vez seleccionado el criterio y los predictores se obtiene una medida de ellos. Para mantener la similitud con el modelo de lógica difusa, los valores irán desde el 1 al 4, en donde el 4 indica que el candidato es excelente en lo que se evalúa, 3 que es bueno, 2 que es regular y 1 que es malo o que simplemente no cumple con el requisito.

Paso 4: Relación del predictor con el criterio. Se determina si existe o no una relación significativa entre el predictor y lo que se pretende predecir.

Paso 5: Aceptar o rechazar los predictores empleados. De acuerdo con los resultados obtenidos en el paso anterior, se decide si se debe aceptar o rechazar los predictores. En caso de que existan correlaciones parciales no significativas se deben observar las correlaciones que hay entre las variables independientes entre sí y con la variable dependiente, luego identificar y emplear solo los predictores que se consideren como los más importantes para explicar la variación en la variable dependiente.

\section{RESULTADOS: EN UNA SECCIÓN}

Resultado de la lógica difusa. A continuación se muestran, parcialmente, los resultados solo para el puesto de Seguridad.

Paso 1: Requisitos exigidos. En la Tabla 2 se muestran los requisitos obligatorios (no compensables) y los requisitos propios del puesto. Así por ejemplo, si el candidato no posee, a lo menos, un nivel de educación Media queda fuera del proceso.

Paso 2: Definición de los coeficientes. En la Tabla 3 están las importancias relativas de cada grupo de requisitos propios del puesto. 
Tabla 2. Requisitos exigidos.

\begin{tabular}{|l|c|}
\hline \multicolumn{2}{|c|}{ Requisitos mínimos } \\
\hline Sección a la que postula & Seguridad \\
\hline Nivel de educación & Media \\
\hline Edad mínima & 18 \\
\hline ¿Está en Dicom? & No \\
\hline Certificado médico & Aprobado \\
\hline Servicio militar & Al día \\
\hline
\end{tabular}

\begin{tabular}{|c|c|c|c|c|}
\hline Requisitos del puesto & \multicolumn{4}{|c|}{ Etiquetas lingüísticas } \\
\hline Habilidades & Exc. & Bue. & Reg. & Malo \\
\hline Proactividad & $\mathrm{X}$ & & & \\
\hline Empatía & $\mathrm{X}$ & & & \\
\hline Conversación y lenguaje & $\mathrm{X}$ & & & \\
\hline
\end{tabular}

\begin{tabular}{|l|c|c|c|c|}
\hline Rasgos generales & Exc. & Bue. & Reg. & Malo \\
\hline Apariencia & & & $\mathrm{X}$ & \\
\hline Actitud & $\mathrm{X}$ & & & \\
\hline Motivación por el trabajo & $\mathrm{X}$ & & & \\
\hline Potencial para el cargo & $\mathrm{X}$ & & & \\
\hline
\end{tabular}

\begin{tabular}{|l|c|c|c|c|}
\hline Exigencias básicas & Exc. & Bue. & Reg. & Malo \\
\hline Examen psicológico & $\mathrm{X}$ & & & \\
\hline Curso OS10 & & $\mathrm{X}$ & & \\
\hline Antecedentes & $\mathrm{X}$ & & & \\
\hline Experiencia laboral & & $\mathrm{X}$ & & \\
\hline Aptitudes físicas & $\mathrm{X}$ & & & \\
\hline
\end{tabular}

Tabla 3. Coeficientes según sección.

\begin{tabular}{|l|c|}
\hline \multicolumn{1}{|c|}{ Requisitos del puesto } & Seguridad \\
\hline Exigencias básicas & $80 \%$ \\
\hline Habilidades & $10 \%$ \\
\hline Rasgos generales & $10 \%$ \\
\hline Total & $\mathbf{1 0 0 \%}$ \\
\hline
\end{tabular}

En este caso se valora más las "Exigencias básicas" que posea el postulante en vez de las "Habilidades" o "Rasgos generales".

Paso 3: Antecedentes de los postulantes. Los datos del postulante 1 se muestran en la Tabla 4. En ella se aprecia que cumple con los requisitos mínimos, y por lo tanto continúa en el proceso.

Tabla 4. Antecedentes de los postulantes.

\begin{tabular}{|l|c|}
\hline \multicolumn{2}{|c|}{ Datos postulante 1 } \\
\hline Nombre & Colaborador 1 \\
\hline Sección a la que postula & Seguridad \\
\hline Nivel de educación & Media \\
\hline Edad & 29 \\
\hline
\end{tabular}

\begin{tabular}{|l|c|}
\hline ¿Está en Dicom? & No \\
\hline Certificado médico & Aprobado \\
\hline Servicio militar & Cumple \\
\hline
\end{tabular}

\begin{tabular}{|l|c|}
\hline ¿Cumple requisitos? & Sí \\
\hline
\end{tabular}

\begin{tabular}{|l|l|}
\hline Requisitos del puesto & Etiquetas lingüísticas \\
\hline
\end{tabular}

\begin{tabular}{|l|c|c|c|c|}
\hline Habilidades & Exc. & Bue. & Reg. & Malo \\
\hline Proactividad & $\mathrm{X}$ & & & \\
\hline Empatía & & $\mathrm{X}$ & & \\
\hline Conversación y lenguaje & $\mathrm{X}$ & & & \\
\hline
\end{tabular}

\begin{tabular}{|l|c|c|c|c|}
\hline Rasgos generales & Exc. & Bue. & Reg. & Malo \\
\hline Apariencia & $\mathrm{X}$ & & & \\
\hline Actitud & & $\mathrm{X}$ & & \\
\hline Motivación por el trabajo & $\mathrm{X}$ & & & \\
\hline Potencial para el cargo & & $\mathrm{X}$ & & \\
\hline
\end{tabular}

\begin{tabular}{|l|c|c|c|c|}
\hline Exigencias básicas & Exc. & Bue. & Reg. & Malo \\
\hline Examen psicológico & & $\mathrm{X}$ & & \\
\hline Curso OS10 & $\mathrm{X}$ & & & \\
\hline Antecedentes & $\mathrm{X}$ & & & \\
\hline Experiencia laboral & & $\mathrm{X}$ & & \\
\hline Aptitudes físicas & & & $\mathrm{X}$ & \\
\hline
\end{tabular}

Pasos 4 y 5: Variables difusas y calificación por grupo de requisitos. Los intervalos de las variables difusas triangulares y con solapamiento del candidato ideal y del candidato 1 están en las Tablas 5 y 6.

Paso 6: Elección del mejor postulante. Una vez ingresados los antecedentes de todos los postulantes se procede a calcular tanto la Distancia de Hamming como el Coeficiente de Adecuación. En este caso la Tabla 7 muestra los resultados de solo dos candidatos. La Distancia de Hamming señala que el mejor para el puesto de seguridad es el $\mathrm{N}^{\mathrm{o}} 1$, ya que tiene la menor distancia; en cambio, el Coeficiente de Adecuación elige al candidato $\mathrm{N}^{\circ} 2$, ya que tiene el mayor valor.

Tabla 5. Variables difusas candidato ideal.

\begin{tabular}{|c|c|}
\hline \multicolumn{2}{|c|}{ EVALUACIÓN CANDIDATO IDEAL } \\
\hline Sección a la que postula & Seguridad \\
\hline
\end{tabular}

\begin{tabular}{|l|l|}
\hline Requisitos del puesto & Etiquetas lingüísticas \\
\hline
\end{tabular}

\begin{tabular}{|c|c|c|c|c|c|}
\hline \multicolumn{2}{|l|}{ Habilidades } & Exc. & Bue. & Reg. & Malo \\
\hline \multicolumn{2}{|l|}{ Proactividad } & 1086 & & & \\
\hline \multicolumn{2}{|l|}{ Empatía } & 1086 & & & \\
\hline \multicolumn{2}{|c|}{ Conversación y lenguaje } & 1086 & & & \\
\hline Calificación & $10,0 \quad 8,00$ & 6,00 & & & \\
\hline
\end{tabular}




\begin{tabular}{|c|c|c|c|c|}
\hline Rasgos generales & Exc. & Bue. & Reg. & Malo \\
\hline Apariencia & & & 642 & \\
\hline Actitud & $\begin{array}{lll}10 & 8 & 6 \\
\end{array}$ & & & \\
\hline Motivación por el trabajo & $10 \begin{array}{lll}10 & 8 & 6 \\
\end{array}$ & & & \\
\hline Potencial para el cargo & $\begin{array}{lll}10 & 8 & 6 \\
\end{array}$ & & & \\
\hline Calificación & $0 \quad 5,00$ & & & \\
\hline
\end{tabular}

\begin{tabular}{|c|c|c|c|c|}
\hline Exigencias básicas & Exc. & Bue. & Reg. & Malo \\
\hline Examen psicológico & 1086 & & & \\
\hline Curso OS10 & & 864 & & \\
\hline Antecedentes & 1086 & & & \\
\hline Experiencia laboral & & 864 & & \\
\hline Aptitudes físicas & 1086 & & & \\
\hline Calificación & $0 \quad 5,20$ & & & \\
\hline
\end{tabular}

Tabla 6. Variables difusas candidato 1.

\begin{tabular}{|c|c|c|c|c|}
\hline \multicolumn{5}{|c|}{ EVALUACIÓN CANDIDATO 1} \\
\hline Sección a la que postula & \multicolumn{4}{|c|}{ Seguridad } \\
\hline Requisitos del puesto & \multicolumn{4}{|c|}{ Etiquetas lingüísticas } \\
\hline Habilidades & Exc. & Bue. & Reg. & Malo \\
\hline Proactividad & 1086 & & & \\
\hline Empatía & & 864 & & \\
\hline Conversación y lenguaje & 1086 & & & \\
\hline Calificación & 5,33 & & & \\
\hline
\end{tabular}

\begin{tabular}{|c|c|c|c|c|}
\hline Rasgos generales & Exc. & Bue. & Reg. & Malo \\
\hline Apariencia & $\begin{array}{lll}10 & 8 & 6\end{array}$ & & & \\
\hline Actitud & & 864 & & \\
\hline Motivación por el trabajo & 1086 & & & \\
\hline Potencial para el cargo & & 864 & & \\
\hline \begin{tabular}{l|ll} 
Calificación & $\mathbf{9 , 0 0} \quad 7,0$ \\
\end{tabular} & $0 \quad 5,00$ & & & \\
\hline
\end{tabular}

\begin{tabular}{|c|c|c|c|c|c|}
\hline \multicolumn{2}{|c|}{ Exigencias básicas } & Exc. & Bue. & Reg. & Malo \\
\hline \multicolumn{2}{|c|}{ Examen psicológico } & & 864 & & \\
\hline \multicolumn{2}{|l|}{ Curso OS10 } & $\begin{array}{llll}10 & 8 & 6 \\
\end{array}$ & & & \\
\hline \multicolumn{2}{|l|}{ Antecedentes } & 1086 & & & \\
\hline \multicolumn{2}{|c|}{ Experiencia laboral } & & 864 & & \\
\hline \multicolumn{2}{|c|}{ Aptitudes físicas } & & & 642 & \\
\hline Calificación & 8,40 & 4,40 & & & \\
\hline
\end{tabular}

Tabla 7. Resultados.

\begin{tabular}{|l|rrr|rrr|}
\hline \multicolumn{1}{|c|}{$\begin{array}{c}\text { Requisitos del } \\
\text { puesto }\end{array}$} & \multicolumn{2}{|c|}{ Perfil ideal } & \multicolumn{2}{l|}{ Postulante 1 } \\
\hline Habilidades & 10,0 & 8,0 & 6,0 & 9,3 & 7,3 & 5,3 \\
Rasgos generales & 9,0 & 7,0 & 5,0 & 9,0 & 7,0 & 5,0 \\
Exigencias básicas & 9,2 & 7,2 & 5,2 & 8,4 & 6,4 & 4,4 \\
\hline Ponderación & 9,3 & 7,3 & 5,3 & 8,6 & 6,6 & 4,6 \\
\hline
\end{tabular}

\begin{tabular}{|l|l|}
\hline Distancia de Hamming & 0,70667 \\
\hline Coeficiente de adecuación & 1,42915 \\
\hline
\end{tabular}

\begin{tabular}{|l|rrr|rrr|}
\hline \multicolumn{1}{|c|}{$\begin{array}{c}\text { Requisitos del } \\
\text { puesto }\end{array}$} & \multicolumn{2}{|c|}{ Perfil ideal } & \multicolumn{2}{|c|}{ Postulante 2 } \\
\hline Habilidades & 10,0 & 8,0 & 6,0 & 8,7 & 6,7 & 4,7 \\
Rasgos generales & 9,0 & 7,0 & 5,0 & 8,5 & 6,5 & 4,5 \\
Exigencias básicas & 9,2 & 7,2 & 5,2 & 7,6 & 5,6 & 3,6 \\
\hline Ponderación & 9,3 & 7,3 & 5,3 & 7,8 & 5,8 & 3,8 \\
\hline
\end{tabular}

\begin{tabular}{|l|l|}
\hline Distancia de Hamming & 1,46333 \\
\hline Coeficiente de adecuación & 2,15375 \\
\hline
\end{tabular}

Resultado de la regresión múltiple. A continuación se muestran, parcialmente, los resultados solo para el puesto de Seguridad.

Paso 1: Análisis del puesto de trabajo. Los requisitos o exigencias importantes para la sección de "Seguridad" son que los colaboradores deben observar y comprobar los sistemas de vigilancia, vigilar y autorizar la entrada y salida de personas al local, mantener el orden y proteger la propiedad, detectar e informar sobre las actividades diarias e irregularidades, entre otras actividades. Por tanto, los postulantes a esta sección deben ser proactivos, empáticos, tener habilidades de comunicación, buena apariencia, actitud y motivación por el trabajo. Además, deben tener aprobado el examen psicológico, haber realizado el curso OS10, tener antecedentes limpios, las aptitudes físicas y cierta experiencia laboral.

Paso 2: Selección de criterios y predictores. Para la sección de "Seguridad", las variables independientes corresponden al promedio ponderado de las valorizaciones que le son asignadas al postulante durante la entrevista en los aspectos señalados en la Tabla 8 .

Paso 3: Medición de criterio y predictores. Se procede a calcular los promedios ponderados para los predictores de la sección de "Seguridad", los que se presentan en la Tabla 9.

Paso 4: Relación del predictor con el criterio. Se determina la ecuación de regresión para cada sección. Para la sección "Seguridad", la ecuación de regresión es:

$\hat{Y}=-0,610+0,247 X_{1}+0,638 X_{2}+0,335 X_{3}$ 
Tabla 8. Predictores de la sección Seguridad.

\begin{tabular}{|l|c|}
\hline \multicolumn{1}{|c|}{ Habilidades } & Ponderación \\
\hline Proactividad & $25 \%$ \\
Empatía & $25 \%$ \\
Conversación y lenguaje & $50 \%$ \\
\hline
\end{tabular}

\begin{tabular}{|l|c|}
\hline \multicolumn{1}{|c|}{ Rasgos generales } & Ponderación \\
\hline Apariencia & $23,33 \%$ \\
Actitud & $23,33 \%$ \\
Motivación por el trabajo & $53,34 \%$ \\
\hline
\end{tabular}

\begin{tabular}{|l|c|}
\hline \multicolumn{1}{|c|}{ Exigencias básicas } & Ponderación \\
\hline Examen psicológico & $82,0 \%$ \\
Curso OS10 & $4,5 \%$ \\
Antecedentes & $4,5 \%$ \\
Experiencia laboral & $4,5 \%$ \\
Aptitudes & $4,5 \%$ \\
\hline
\end{tabular}

Paso 5: Aceptar o rechazar los predictores empleados. Para excluir una o más variables independientes en la ecuación se utilizará el procedimiento "Regresión paso a paso" (stepwise regression), con ello, para la sección "Seguridad", finalmente se trabajará solo con dos predictores: "Empatía" y "Experiencia Laboral”, con ello la ecuación de regresión es:

$$
\hat{Y}=0,525+0,559 X_{1}+0,348 X_{2}
$$

$\mathrm{Al}$ reemplazar los valores en la ecuación se obtienen los resultados pronosticados $(\hat{Y})$ para cada postulante. Entonces, de estos valores se elegirá como al mejor candidato el postulante que obtenga el mayor puntaje, siendo para este caso los postulantes número $9 \mathrm{y}$ 20, como se puede observar en la Tabla 10:

\section{RESULTADOS: EN TODAS LAS SECCIONES}

Resultados obtenidos con el modelo de regresión múltiple. El modelo de regresión múltiple no se pudo aplicar en cuatro secciones: "Frutas y Verduras", "Fiambrería y Deli", "Consumible" y "Carnes y Pescados", debido que en estas secciones el tamaño muestral de postulantes no es suficiente para ser aplicado con este modelo. Es decir, este modelo no se puede aplicar a un puesto de trabajo cuando la cantidad de postulantes es pequeña.

Como se puede observar en la Tabla 11 el coeficiente de determinación $\left(\mathrm{R}^{2}\right)$ en todos los casos es alto,

Tabla 9. Criterios y predictores de la sección seguridad.

\begin{tabular}{|c|c|c|c|c|c|c|c|c|c|c|c|c|c|c|c|}
\hline \multirow[b]{3}{*}{ 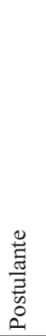 } & \multicolumn{4}{|c|}{ HABIIDADES } & \multicolumn{4}{|c|}{ RASGOS GENERALES } & \multicolumn{6}{|c|}{ EXIGENCIAS BÁSICAS } & \multirow{2}{*}{ 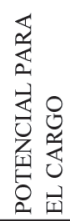 } \\
\hline & 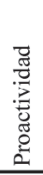 & 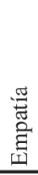 & 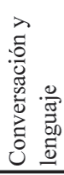 & & $\begin{array}{l}\frac{\pi}{0} \\
\frac{0}{0} \\
\frac{0}{\pi} \\
\frac{2}{4}\end{array}$ & 总 & 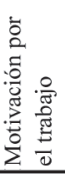 & & 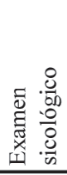 & $\begin{array}{l}0 \\
0 \\
0 \\
0 \\
\vdots \\
0 \\
0\end{array}$ & 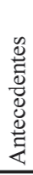 & 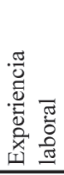 & 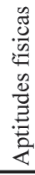 & & \\
\hline & $\begin{array}{l}\text { ठें } \\
\text { in } \\
\text { in }\end{array}$ & $\begin{array}{l}\dot{\sigma}^{\circ} \\
\ddot{c}\end{array}$ & $\begin{array}{l}\text { oें } \\
\text { iे } \\
\text { in }\end{array}$ & $\mathrm{X}_{1}$ & 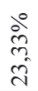 & ले & $\begin{array}{l}\stackrel{\circ}{\mathrm{j}} \\
\mathrm{m}_{n}^{2}\end{array}$ & $\mathrm{X}_{2}$ & $\begin{array}{l}\text { 今े } \\
\text { ठ } \\
\text { o. }\end{array}$ & $\begin{array}{l}\stackrel{0}{\circ} \\
\dot{n} \\
\text { f }\end{array}$ & $\begin{array}{l}\text { 今े } \\
\text { ñ } \\
\text { f }\end{array}$ & $\begin{array}{l}\stackrel{\circ}{0} \\
\stackrel{n}{+}\end{array}$ & $\begin{array}{l}\text { 足 } \\
\text { n. } \\
+\end{array}$ & $\mathrm{X}_{3}$ & Y \\
\hline 1 & 4 & 3 & 4 & 3,75 & 4 & 3 & 4 & 3,77 & 3 & 4 & 4 & 3 & 2 & 3,05 & 3 \\
\hline 2 & 3 & 3 & 4 & 3,50 & 3 & 4 & 3 & 3,23 & 2 & 3 & 4 & 2 & 3 & 2,18 & 3 \\
\hline 3 & 3 & 3 & 4 & 3,50 & 3 & 3 & 3 & 3,00 & 3 & 3 & 4 & 2 & 3 & 3,00 & 3 \\
\hline 4 & 3 & 3 & 3 & 3,00 & 3 & 4 & 4 & 3,77 & 3 & 3 & 4 & 4 & 3 & 3,09 & 4 \\
\hline 5 & 2 & 2 & 3 & 2,50 & 3 & 3 & 2 & 2,47 & 3 & 2 & 4 & 3 & 3 & 3,00 & 2 \\
\hline 6 & 3 & 3 & 3 & 3,00 & 3 & 4 & 4 & 3,77 & 3 & 3 & 4 & 3 & 3 & 3,05 & 3 \\
\hline 7 & 2 & 2 & 2 & 2,00 & 3 & 3 & 1 & 1,93 & 2 & 2 & 4 & 1 & 2 & 2,05 & 2 \\
\hline 8 & 3 & 3 & 2 & 2,50 & 3 & 2 & 3 & 2,77 & 3 & 3 & 4 & 3 & 3 & 3,05 & 3 \\
\hline 9 & 3 & 4 & 4 & 3,75 & 3 & 4 & 4 & 3,77 & 3 & 3 & 4 & 4 & 3 & 3,09 & 4 \\
\hline 10 & 3 & 3 & 3 & 3,00 & 3 & 2 & 3 & 2,77 & 3 & 2 & 4 & 3 & 3 & 3,00 & 3 \\
\hline 11 & 3 & 3 & 4 & 3,50 & 3 & 3 & 4 & 3,53 & 3 & 3 & 4 & 4 & 3 & 3,09 & 4 \\
\hline 12 & 3 & 3 & 3 & 3,00 & 3 & 3 & 3 & 3,00 & 3 & 3 & 4 & 3 & 3 & 3,05 & 4 \\
\hline 13 & 3 & 3 & 4 & 3,50 & 4 & 4 & 3 & 3,47 & 3 & 3 & 4 & 4 & 3 & 3,09 & 4 \\
\hline 14 & 3 & 3 & 3 & 3,00 & 3 & 3 & 3 & 3,00 & 3 & 3 & 4 & 4 & 3 & 3,09 & 3 \\
\hline 15 & 2 & 2 & 3 & 2,50 & 3 & 3 & 3 & 3,00 & 3 & 3 & 4 & 3 & 3 & 3,05 & 3 \\
\hline 16 & 3 & 2 & 2 & 2,25 & 3 & 2 & 3 & 2,77 & 3 & 2 & 4 & 3 & 3 & 3,00 & 3 \\
\hline 17 & 2 & 1 & 3 & 2,25 & 3 & 2 & 2 & 2,23 & 3 & 3 & 4 & 3 & 3 & 3,05 & 2 \\
\hline 18 & 3 & 3 & 3 & 3,00 & 3 & 3 & 2 & 2,47 & 3 & 3 & 4 & 3 & 3 & 3,05 & 3 \\
\hline 19 & 3 & 3 & 2 & 2,50 & 3 & 3 & 3 & 3,00 & 3 & 2 & 4 & 2 & 3 & 2,96 & 3 \\
\hline 20 & 4 & 4 & 4 & 4,00 & 4 & 4 & 4 & 4,00 & 3 & 3 & 4 & 4 & 4 & 3,14 & 4 \\
\hline
\end{tabular}


Tabla 10. Criterio, predictores y pronóstico para la sección Seguridad.

\begin{tabular}{|c|c|c|c|c|}
\hline Postulante & Empatía & $\begin{array}{c}\text { Experiencia } \\
\text { laboral } \\
\mathbf{X}_{\mathbf{2}}\end{array}$ & $\begin{array}{c}\text { Potencial } \\
\text { para el } \\
\text { cargo } \\
\mathbf{Y}\end{array}$ & $\hat{\mathbf{Y}}$ \\
\hline 1 & 3 & 3 & 3 & 3,25 \\
\hline 2 & 3 & 2 & 3 & 2,90 \\
\hline 3 & 3 & 2 & 3 & 2,90 \\
\hline 4 & 3 & 4 & 4 & 3,59 \\
\hline 5 & 2 & 3 & 2 & 2,69 \\
\hline 6 & 3 & 3 & 3 & 3,25 \\
\hline 7 & 2 & 1 & 2 & 1,99 \\
\hline 8 & 3 & 3 & 3 & 3,25 \\
\hline 9 & 4 & 4 & 4 & 4,15 \\
\hline 10 & 3 & 3 & 3 & 3,25 \\
\hline 11 & 3 & 4 & 4 & 3,59 \\
\hline 12 & 3 & 3 & 4 & 3,25 \\
\hline 13 & 3 & 4 & 4 & 3,59 \\
\hline 14 & 3 & 4 & 3 & 3,59 \\
\hline 15 & 2 & 3 & 3 & 2,69 \\
\hline 16 & 2 & 3 & 3 & 2,69 \\
\hline 17 & 1 & 3 & 2 & 2,13 \\
\hline 18 & 3 & 3 & 3 & 3,25 \\
\hline 19 & 3 & 2 & 3 & 2,90 \\
\hline 20 & 4 & 4 & 4 & 4,15 \\
\hline & & & & \\
\hline
\end{tabular}

Tabla 11. Resultados del modelo de regresión múltiple.

\begin{tabular}{|l|c|c|}
\hline \multicolumn{1}{|c|}{ Sección } & $\mathbf{R}^{\mathbf{2}}$ & $\begin{array}{c}\text { Mejor } \\
\text { postulante }\end{array}$ \\
\hline $\begin{array}{l}\text { Seguridad: } \\
\hat{Y}=0,525+0,559 \mathrm{X}_{1}+0,348 \mathrm{X}_{2}\end{array}$ & $71,0 \%$ & $\mathrm{~N}^{\circ} 9 \mathrm{y} 20$ \\
\hline $\begin{array}{l}\text { Hardlines: } \\
\hat{Y}=-3,038+1,308 \mathrm{X}_{1}+0,769 \mathrm{X}_{2}\end{array}$ & $89,5 \%$ & $\mathrm{~N}^{\circ} 1$ \\
\hline $\begin{array}{l}\text { Entretenimiento: } \\
\hat{Y}=0,188+0,563 \mathrm{X}_{1}+0,375 \mathrm{X}_{2}\end{array}$ & $90,0 \%$ & $\mathrm{~N}^{\circ} 2$ \\
\hline $\begin{array}{l}\text { Panadería y Pastelería: } \\
\hat{Y}=-1,036+0,643 \mathrm{X}_{1}+0,714 \mathrm{X}_{2}\end{array}$ & $89,0 \%$ & $\mathrm{~N}^{\circ} 12$ \\
\hline $\begin{array}{l}\text { Vestuario y Mundo Bebé: } \\
\hat{Y}=0,043+0,543 \mathrm{X}_{1}+0,348 \mathrm{X}_{2}\end{array}$ & $80,3 \%$ & $\mathrm{~N}^{\circ} 1,4$ y 8 \\
\hline
\end{tabular}

es decir, que los predictores explican bastante a la variable dependiente. Cuando el modelo arroja que existe más de un candidato ideal, será necesario agregar otros requisitos específicos para quedar con un solo candidato, o bien, reponer una variable que haya sido descartada por su bajo poder explicativo pero que podría permitir que quede un único candidato.

Resultados obtenidos con el modelo basado en Lógica Difusa. En la Tabla 12 se dan a conocer los resultados obtenidos al emplear el modelo de selección de personal basado en la lógica difusa para todas las secciones.

Tabla 12. Tabla resumen del modelo basado en lógica difusa

\begin{tabular}{|l|c|c|c|c|}
\hline \multicolumn{1}{|c|}{ Sección } & $\begin{array}{c}\text { Distancia } \\
\text { de } \\
\text { Hamming }\end{array}$ & $\begin{array}{c}\text { Mejor } \\
\text { postulante }\end{array}$ & $\begin{array}{c}\text { Coeficiente } \\
\text { de } \\
\text { adecuación }\end{array}$ & $\begin{array}{c}\text { Mejor } \\
\text { postulante }\end{array}$ \\
\hline Seguridad & 0,1 & $\mathrm{~N}^{\circ} 20$ & 1,0512 & $\mathrm{~N}^{\mathrm{o}} 20$ \\
\hline Hardlines & 0,5867 & $\mathrm{~N}^{\mathrm{o}} 5$ & 1,3438 & $\mathrm{~N}^{\mathrm{o}} 5$ \\
\hline $\begin{array}{l}\text { Frutas y } \\
\text { Verduras }\end{array}$ & 0 & $\mathrm{~N}^{\mathrm{o}} 3$ & 1 & $\mathrm{~N}^{\mathrm{o}} 3$ \\
\hline $\begin{array}{l}\text { Fiambrería } \\
\text { y Deli }\end{array}$ & 0,08 & $\mathrm{~N}^{\mathrm{o}} 1$ & 1,0408 & $\mathrm{~N}^{\mathrm{o}} 1$ \\
\hline $\begin{array}{l}\text { Entreteni- } \\
\text { miento }\end{array}$ & 0,2378 & $\mathrm{~N}^{\mathrm{o}} 6$ & 1,1264 & $\mathrm{~N}^{\mathrm{o}} 6$ \\
\hline Consumible & 0,1071 & $\mathrm{~N}^{\mathrm{o}} 4$ & 1,0550 & $\mathrm{~N}^{\mathrm{o}} 4$ \\
\hline $\begin{array}{l}\text { Carnes y } \\
\text { Pescados }\end{array}$ & 0,3543 & $\mathrm{~N}^{\mathrm{o}} 5$ & 1,1944 & $\mathrm{~N}^{\mathrm{o}} 5$ \\
\hline $\begin{array}{l}\text { Panadería y } \\
\text { Pastelería }\end{array}$ & 0,05 & $\mathrm{~N}^{\mathrm{o}} 2 \mathrm{y} 6$ & 1,0253 & $\mathrm{~N}^{\mathrm{o}} 2$ y 6 \\
\hline $\begin{array}{l}\text { Vestuario } \\
\text { y Mundo } \\
\text { bebé }\end{array}$ & 0,4667 & $\mathrm{~N}^{\mathrm{o}} 5 \mathrm{y} 7$ & 1,2642 & $\mathrm{~N}^{\mathrm{o}} 5 \mathrm{y} 7$ \\
\hline
\end{tabular}

La utilización de etiquetas lingüísticas y la operativa con ellas no entregan un candidato ideal para un puesto, sino que para un mismo puesto habrá varios candidatos idóneos con distintos grados de verosimilitud. Cuando dos candidatos son idóneos con un grado similar de verosimilitud, será necesario agregar otros requisitos específicos para quedar con un solo candidato.

Al observar la Tabla 12 se puede apreciar que el método Distancia de Hamming como el de Coeficiente de Adecuación arrojan los mismos postulantes como los mejores candidatos en todas las secciones. Además, se observa que en la sección de "Frutas y verduras" la puntuación obtenida en la distancia de Hamming y en Coeficiente de Adecuación es de 0 y 1 , respectivamente, lo que indica que el postulante seleccionado posee un alto grado de verosimilitud.

Resumen. En la Tabla 13 se muestra un resumen, para cada sección, del candidato elegido por ambos modelos y se comparan con la evaluación de desempeño del mejor trabajador (una vez contratado).

En la Tabla 13 se aprecia que el modelo de regresión múltiple de las cinco secciones evaluadas acertó en 
Tabla 13. Mejor postulante según los modelos.

\begin{tabular}{|c|c|c|c|}
\hline Sección & $\begin{array}{l}\text { Regres. } \\
\text { múltip. }\end{array}$ & $\begin{array}{l}\text { Lógica } \\
\text { difusa }\end{array}$ & $\begin{array}{c}\text { Eval. de } \\
\text { desempeño }\end{array}$ \\
\hline Seguridad & $\mathrm{N}^{\circ} 9$ y 20 & $\mathrm{~N}^{\circ} 20$ & $\mathrm{~N}^{\mathrm{o}} 20$ \\
\hline Hardlines & $\mathrm{N}^{\mathrm{o}} 1$ & $\mathrm{~N}^{\circ} 5$ & $\mathrm{~N}^{\mathrm{o}} 1$ \\
\hline Frutas y Verduras & - & $\mathrm{N}^{\mathrm{o}} 3$ & $\mathrm{~N}^{\mathrm{o}} 1$ \\
\hline Fiambrería y Deli & - & $\mathrm{N}^{\mathrm{o}} 1$ & $\mathrm{~N}^{\circ} 3$ \\
\hline Entretenimiento & $\mathrm{N}^{\mathrm{o}} 2$ & $\mathrm{~N}^{\mathrm{o}} 6$ & $\mathrm{~N}^{\circ} 4$ \\
\hline Consumible & - & $\mathrm{N}^{\circ} 4$ & $\mathrm{~N}^{\mathrm{o}} 2$ \\
\hline Carnes y Pescados & - & $\mathrm{N}^{\circ} 5$ & $\mathrm{~N}^{\circ} 5$ \\
\hline Panadería y Pastelería & $\mathrm{N}^{\mathrm{o}} 12$ & $N^{\circ} 2$ y 6 & $\mathrm{~N}^{\mathrm{o}} 12$ \\
\hline Vestuario y Mundo Bebé & $\begin{array}{c}\mathrm{N}^{\circ} 1,4 \\
\text { y } 8\end{array}$ & $\mathrm{~N}^{\circ} 5$ y 7 & $\mathrm{~N}^{\mathrm{o}} 1$ \\
\hline
\end{tabular}

4 (Seguridad; Hardlines; Panadería y Pastelería; y Vestuario y Mundo Bebé) lo que representa un $80 \%$ de acierto. En cambio, el modelo de lógica difusa, de las 9 secciones, solo acertó en dos de ellas (Seguridad y Carnes y Pescados), lo que representa $22 \%$.

\section{CONCLUSIONES}

Actualmente los recursos humanos se consideran un activo fuente de ventaja competitiva para la empresa. Una manera de aprovechar lo mejor posible este recurso es mediante la gestión por competencias. Las competencias son los conocimientos, habilidades, actitudes, aptitudes, etc., que hacen que el desarrollo de ciertas tareas y actividades, así como el logro de determinados resultados, sean sobresalientes.

El objetivo de este trabajo era elaborar un modelo de selección de personal aplicando la Lógica Difusa y compararla con un modelo clásico de regresión múltiple, de modo de determinar cuál de ellos es más preciso al momento de seleccionar un postulante a un determinado puesto. Para esto fue necesario comparar los postulantes elegidos con las últimas evaluaciones realizadas por la empresa a todos sus empleados. Si en cada sección el empleado mejor evaluado coincide con el candidato elegido por uno de los modelos, entonces se considera como un acierto del modelo.

En general, aunque es recomendable que las etiquetas lingüísticas se definan en número impar para alcanzar una cierta simetría en la evaluación, se prefirió usar la metodología sugerida en [7] y [4], considerando solo cuatro etiquetas. Como ello se aplicó en ambos modelos, los resultados obtenidos son homogéneos y comparables.

Si bien los resultados muestran que el modelo optado utilizando variables difusas triangulares y con solapamiento de $25 \%$ del área es ampliamente superado por el modelo de regresión múltiple, sería recomendable probar otros modelos de lógica difusa (por ejemplo, con distintas funciones de pertenencias, con distintos porcentajes de solapamiento).

Una posible distorsión en los resultados puede ocurrir con "postulantes" (actualmente empleados) que han ingresado a la empresa en distintos años, y al compararlos con la última evaluación de desempeño se podrían ver favorecidos, ya que los trabajadores más antiguos, por el hecho de tener mayor experiencia, podrían hoy tener mejor evaluación. En un futuro trabajo sería recomendable que solo se considerasen "postulantes" con antigüedades similares en un mismo puesto.

\section{REFERENCIAS}

[1] S. Aja. "Un nuevo marco matricial para la implementación eficiente de esquemas de inferencia difusa aplicados al procesado de información no numérica”. Biblioteca Virtual Miguel de Cervantes. Alicante, España. 2005.

[2] G. Bojadzvol and M. Bojadziev. "Fuzzy Logic For Business, Finance, and Management". Worls Scientific Publishing Co. Pte. Ltd. 2a Edition. 2007.

[3] G. Bohlander, S. Snell y A. Sherman. "Administración de recursos humanos". Editorial Thomson Learning. 12va edición. 2004.

[4] A. Caballero, A. Gento y A. Redondo. "Selección de personal utilizando lógica borrosa". IX Congreso de Ingeniería de Organización. Gijón, España. 2005

[5] M. Caetano, D. Gherardi and T. Yoneyama. "Applied Engineering on Biosystems: Representation of policies aimed at the reduction of global warming using fuzzy rules". Proceedings of the WSEAS International Conference on Mathematical and Computational Methods in Science and Engineering (MACMESE'09). Baltimore, MD, USA. November, 2009.

[6] L. Canós and V. Liern. "Soft computingbased aggregation methods for human 
resource management". European Journal of Operational Research. Vol. 189, Issue 1, pp. 669-681. 2008.

[7] V. Cantillana. "Selección de personal, en condiciones de incertidumbre, aplicando la lógica difusa". Tesis para optar al grado de Magíster en Ingeniería en Gestión y Tecnología. Universidad de Tarapacá. Arica, Chile. 2010.

[8] A. Cuadra. "Un modelo matemático de selección de personal basado en validez concurrente". Revista de Psicología del Trabajo y de las Organizaciones. Vol. 6 No 17, pp. 131-138. 1990.

[9] A. de Ansorena. "15 Pasos para la Selección de Personal con Éxito". Editorial Paidós Ibérica Ediciones S.A. Barcelona, España. 2003.

[10] G. Ferris, S.D. Rosen and D.T. Barnum. "Handbook of Human Resources Management". Blackwell Publishers. 1995.

[11] J. García, J. Medel, J. Sánchez y A. Tequíanez. "Sistemas con Lógica Difusa". Instituto Politécnico Nacional. México. 2009.

[12] J. Gil-Aluja. "La gestión interactiva de los Recursos Humanos en la incertidumbre". Editorial Ceura. Madrid, España. 1996.

[13] C. Huapaya, F. Lizarralde y M. Arona "Modelo basado en Lógica Difusa para el Diagnóstico Cognitivo del Estudiante”. Formación Universitaria. Vol. $5 \mathrm{~N}^{\circ}$ 1, pp. 13-20. 2012.

[14] J. Klir, U. St Clair and B. Yuan. "Fuzzy set theory: foundations and applications". Editorial Prentice Hall. 1997.

[15] F. Mendoza. "Redes neuronales y lógica difusa en la predicción del crecimiento de una matrícula estudiantil - docente". Tesis de grado para optar al título de Licenciatura en Informática mención Ingeniería de Sistemas Informáticos. Universidad Mayor de San Andrés. La Paz, Bolivia. 2007.
[16] A. Mínguez. "Dirección Práctica de Recursos Humanos". Esic Editorial. 2a edición, pp. 58-59. España. 2005.

[17] M. Montes y P. González. "Selección de Personal: La Búsqueda del Candidato Adecuado". Ideaspropias Editorial. 1a edición. España. 2006.

[18] R. Pérez. "Procesado y optimización de espectros Raman mediante técnicas de lógica difusa: aplicación a la identificación de materiales pictóricos". Tesis para optar al grado de doctor. Universidad Politécnica de Cataluña. España. 2005.

[19] M. Porret. "Recursos Humanos, dirigir y gestionar personas en las organizaciones". ESIC Editorial. 3a edición. España. 2008.

[20] J. Ríos. "Análisis y diseño de controladores basados en lógica difusa". Tesis de Grado para obtener el título de Ingeniero en Electrónica. Facultad de Ingeniería. Universidad de San Carlos. Guatemala. 2005.

[21] L. Schnitman and T. Yoneyama. "TakagiSugeno-Kang fuzzy structures in dynamic system modeling". Proceedings of the IASTED International Conference on Control and Application (CA'2001), pp. 160-165. Banff, Canada. June, 2001.

[22] L. Torres, C. Vasconcelos and L. Schnitman. "Exact linearization and fuzzy logic applied to the Control of a magnetic levitation system". Proceedings of the WCCI 2010 IEEE World Congress on Computational Intelligence. Barcelona, España. July, 2010.

[23] E. Trillas y J. Gutiérrez. "Aplicaciones de la lógica borrosa". Editado por Consejo Superior de Investigaciones Científicas. Madrid, España. 1992.

[24] D. Ulrich y G. Zadunaisky. "Recursos humanos champions". Ediciones Granica S.A. 1997.

[25] L. Zadeh. "Fuzzy sets". Information and control. Vol. 8, pp. 338-353. June, 1965. 